\title{
High-resolution Ultrasound Imaging with Unified Pixel-Based Beamforming
}

\author{
Nghia Q. Nguyen and Richard W. Prager
}

\begin{abstract}
This paper describes the development and evaluation of a new beamforming strategy based on pixel-based focusing for ultrasound linear array systems. We first implement conventional pixel-based beamforming in which the transmitted wave is assumed as spherical and diverging from the centre of the transmit subaperture. This assumed wave-shape is only valid within a limited angle on each side of the beam and this restricts the number of different subaperture positions from which data can be combined to improve image quality. By analyzing the field patterns, we propose a new unified pixel-based beamforming algorithm that better adapts to the non-spherical wave-shape of the transmit beam. This approach enables us to select the best-possible signal from each transducer waveform for data superposition. In simulations and a phantom study, we show that the unified pixel-based beamformer offers significant improvements in image quality compared to other delay-and-sum methods but at a higher computational cost. The new algorithm also demonstrates robust performance in a limited in vivo study. Overall, the results show that it is potentially of value in clinical applications.
\end{abstract}

Index Terms - ultrasound imaging, pixel-based beamforming, time delay, high-resolution, image quality.

\section{INTRODUCTION}

Ultrasound imaging beamformer design involves determining the pulse-echo insonification and reconstruction procedure to display useful diagnostic information [1]. The goal is to generate a highly focused beam over the entire imaging region [2]. The most popular beamformer is still conventional dynamic focusing. This method forms the envelope image lineby-line using a fixed transmit focus and a dynamic delay-andsum (DAS) receive focus. During scan conversion the envelope image is interpolated to create virtual scanlines between the actual scanlines. This interpolation does not increase the information content of the image but enhances its perceived smoothness. The image resolution is only optimal at the transmit focal depth [3].

With developments in data storage and real-time computation, several alternative approaches to beamforming have been developed. Two such methods are synthetic aperture

Copyright (c) 2010 IEEE. Personal use of this material is permitted. However, permission to use this material for any other purposes must be obtained from the IEEE by sending a request to pubs-permissions@ieee.org.

Manuscript received May 08, 2015; revised July 06, 2015; accepted July 08, 2015. Asterisk indicates corresponding author.

*N. Q. Nguyen is with the Information Engineering Division, Department of Engineering, University of Cambridge, Cambridge CB2 1PZ, UK (e-mail: nqn20@cam.ac.uk).

R. W. Prager is with the Information Engineering Division, Department of Engineering, University of Cambridge, Cambridge CB2 1PZ, UK (e-mail: rwp@eng.cam.ac.uk)
(SA) [4]-[8] and parallel beamforming [9], [10]. Synthetic aperture imaging involves transmitting a spherical wave with a single element and receiving on all the elements of the transducer. The data thus acquired enables two-way dynamic focusing for each imaging point [4]. A major problem of the SA approach is poor penetration because of the weak transmit power of a single element. Hence, a multi-element subaperture is frequently used to emulate the spherical radiation pattern of a high-power single transmitter [5]. Usually a diverging or plane wave beam is used [8]. Parallel beamforming also employs a broad transmit beam so that several scanlines can be generated within the boundary of each transmit beam. This is often used when a high frame rate is required, even at some loss of spatial resolution.

There is also a family of algorithms, known as pixel-based focusing, that perform dynamic focusing at the pixel level with a focused transmit beam [11]-[16]. They aim at improving spatial resolution by reducing artefacts caused by interpolation [11]-[13] or parallel received beams [14]. In its simplest form, pixel-based focusing generates data for each virtual scanline using echo signals from the nearest transmit cycle [11], [12]. Attempts to accumulate data from a large number of transmit cycles lead to the use of a virtual source approach. In this approach, the source is assumed to be at the focal point and to transmit spherical waves back and forth within a limited angle [17], [18]. Imaging points outside the angle are considered as invalid and discarded [15], [19]. The method was found to improve the spatial resolution and penetration in the outof-focus regions. However, there were also reported artefacts around the focal depth because of the discontinuity in the spherical-wave approximation [15]. To avoid the artefacts, Kim et al. proposed the $S A$ - bidirectional pixel-based focusing (SA-BiPBF) where the transmit focal depth is set outside the imaging region [16]. They achieved smooth reconstructed images with improvements in spatial resolution. However this strategy broadens the transmit beam which may reduce the benefit from using the virtual source.

In this study, we explore new techniques for pixel-based focusing applied to linear array systems. Our goal is to find ways of computing the most accurate high-resolution reconstruction based on a conventional architecture, provided that we have access to all the low-level data and can integrate it in an arbitrarily complex algorithm. We use the term pixel-based beamforming to denote the family of algorithms that employ compounding at individual pixels to increase the information content of the reconstructed image. We develop a geometric analysis to understand the propagation of the outward going ultrasound beam. From this analysis, we design a new dynamic 
calculation for the beam propagation time delay. It selects the relevant backscattered echo signals to restore the wavefront coherently at individual imaging pixels. We name the new method unified pixel-based beamforming.

The remainder of the paper is organized as follows. Section II presents the analysis with accompanying numerical validation using Field II [20], [21]. Both new and existing approaches are evaluated on in vitro and in vivo data acquired using an instrumented ultrasound machine [22] in Section III. Resolution and penetration results with the unified pixel-based beamformer are encouraging and competitive at all depths of the reconstructed image. However, the algorithm comes at a high computational cost. Some broader perspectives on the results are discussed in Section IV followed by final conclusions drawn in Section V.

\section{THEORY AND BEAMFORMER DESIGN}

In the scope of this study, all beamformers use time delay calculations to collect relevant signals to enable data superposition at each imaging pixel. The calculations are based on a geometrical optics approximation which describes the acoustic wave propagation in terms of rays [23].

\section{A. Conventional pixel-based beamforming}

In conventional pixel-based (PB) beamforming, the time delay $\tau_{p}(i, j)$ for an ultrasonic wave generated from transmit $i$ propagating to any imaging point $\mathrm{P}$ and back to element $j$ is calculated as

$$
\tau_{p}(i, j)=\frac{\left|\mathbf{x}_{i}-\mathbf{x}_{p}\right|}{c}+\frac{\left|\mathbf{x}_{j}-\mathbf{x}_{p}\right|}{c}
$$

where $c$ is the sound-speed, $\mathbf{x}_{i}, \mathbf{x}_{j}$, and $\mathbf{x}_{p}$ respectively denote position vectors of the subaperture center of transmit $i$, receiving element $j$, and $\mathrm{P}$ [7]. Data at $\mathrm{P}$ is calculated using

$$
g\left(\mathbf{x}_{p}\right)=\sum_{i}^{N_{t}} \sum_{j}^{N_{r}} w_{i, j} r_{i, j}\left(\tau_{p}(i, j)\right)
$$

where $N_{t}$ is the number of transmits, $N_{r}$ is the number of receiving elements per transmit, $r_{i, j}(t)$ is the waveform or echo RF trace received on element $j$ with transmit $i$, and the $w_{i, j}$ 's are weighting coefficients. This scheme is applied to all points of the imaging region. In the simplest version, $N_{t}=1$ and $r_{i, j}(t)$ is data acquired with the transmit beam closest to $\mathrm{P}$ [11], [12].

The performance of the beamformer depends on appropriate calculation of the transmit time delays between the excitation from the probe and the point where the wave is back-scattered by the target. In the following sections, we analyze the pressure field to show the limitations of some ways of calculating transmit delays. This analysis also helps us develop new time delay calculations to overcome these limitations.

\section{B. Pressure field analysis}

The pressure field from a 1-D linear array has been studied numerically with some simplifying approximations [24], [25]. In this paper, we also develop our analysis based on a numerical method. However, we briefly review and derive some analytical results at a basic level which give insight into the structure of the pressure fields and serve as a guide for the simulations in the next section.

We assume the impulse responses of the transducer elements are identical and the same in every direction. In general, the impulse responses are not the same because of differences caused by directivity patterns, steering and apodization. By taking the transmit delay into account, we model the subaperture as a continuous uniform aperture focused at depth $d$, shown in Figs. 1(a)-(c). A treatment for a discrete aperture can be found in [26].

From the focal point $\mathrm{F}$, we divide the imaging plane into four regions, from (I) to (IV), by using the limited angle $\alpha$ in the virtual source approach [17], [18]. The source is assumed at $\mathrm{F}$ and the transmitted wave is approximated as spherical in regions (I) and (III). In regions (II) and (IV), this approximation cannot be made. Figures. 1(a)-(c) describe three different scenarios of the imaging point $\mathrm{P}$ with respect to the limited angle. Points in region (IV) are treated the same as those in (II). For convenience, we use the same notation in the three figures. The pressure field at imaging point $\mathrm{P}$ can be obtained through the spatiotemporal impulse response, $h\left(\mathbf{x}_{p}, t\right)$, which characterizes the radiation field for a particular transducer geometry [27]. With a sinusoidal and uniform excitation $v(t)$, we can obtain $p\left(\mathbf{x}_{p}, t\right)$ as

$$
p\left(\mathbf{x}_{p}, t\right)=\rho v(t) * \frac{\partial h\left(\mathbf{x}_{p}, t\right)}{\partial t},
$$

where $\rho$ is the equilibrium density of the weakly-scattering medium. With a 1-D solid aperture, $h\left(\mathbf{x}_{p}, t\right)$ is given by (see [27], Equation 3.25)

$$
h\left(\mathbf{x}_{p}, t\right)=\frac{1}{2 \pi} \int_{R_{\min }}^{R_{\max }} \beta(R) \delta\left(t-\frac{R}{c}\right) d R,
$$

where $R$ is the distance from $\mathrm{P}$ to each element of the aperture $S, c$ is the speed of sound, and $\beta(R)$ is the corresponding angle in polar coordinates. We assume the aperture has uniform apodization and ignore it in the integral.

In the simplest case for $\mathrm{P}$ in region (II) (Fig. 1(b)), $R$ monotonically increases from $R_{1}$ to $R_{2}$ at the two ends of the aperture. By changing the variable $t^{\prime}=R / c$ and taking the derivative of (4), we obtain two mathematical pulses for $\partial h\left(\mathbf{x}_{p}, t\right) / \partial t$ :

$$
\frac{\partial h\left(\mathbf{x}_{p}, t\right)}{\partial t}=\frac{c}{2 \pi}\left[\beta_{1} \delta\left(t-\frac{R_{1}}{c}\right)-\beta_{2} \delta\left(t-\frac{R_{2}}{c}\right)\right],
$$

where $\beta_{1}$ and $\beta_{2}$ are angular coefficients associated with $R_{1}$ and $R_{2}$. Substituting (5) into (3), we obtain the pressure field as

$$
p\left(\mathbf{x}_{p}, t\right)=\frac{\rho c}{2 \pi}\left[\beta_{1} v\left(t-\frac{R_{1}}{c}\right)-\beta_{2} v\left(t-\frac{R_{2}}{c}\right)\right],
$$

Thus, $p\left(\mathbf{x}_{p}, t\right)$ is a combination of two delayed versions of the excitation pulse corresponding to distances $R_{1}$ and $R_{2}$. The time delays for these pulses can be calculated straightforwardly given the sound-speed $c$. By modeling the subaperture as a convex arc, however, there is a time lag, $\tau_{0}=\left(R_{0}-d\right) / c$, 


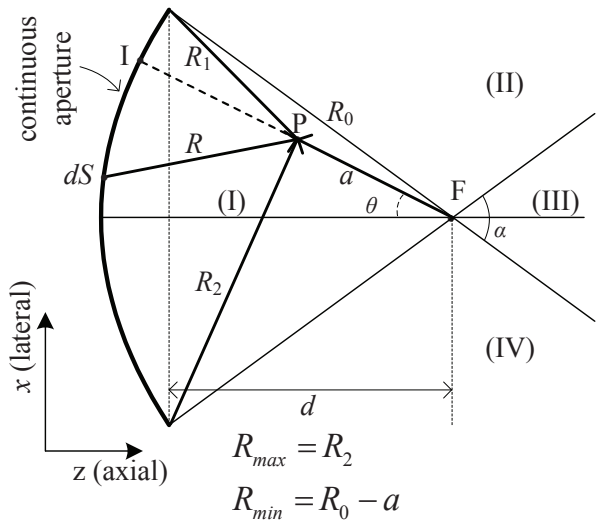

(a)

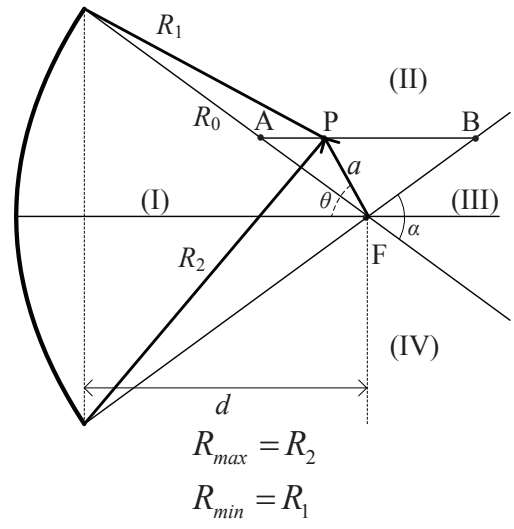

(b)

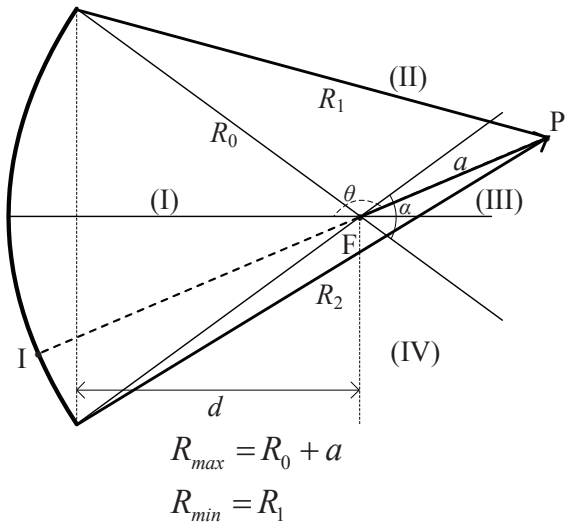

(c)

Fig. 1. Geometries for calculating the pressure field patterns transmitted from a 1-D linear array and observed at individual imaging points. The transmit delays associated with the elements of the active linear aperture are represented by the curvature of the aperture in the axial-lateral plane.

for each calculation because the clock starts counting at the time the last element at the center is fired. The transmit time delays for the two pulses are therefore given by

$$
\tau_{p, 1}=\frac{R_{1}}{c}-\tau_{0} \text { and } \tau_{p, 2}=\frac{R_{2}}{c}-\tau_{0} .
$$

For $\mathrm{P}$ in region (I) (Fig. 1(a)), the minimal distance, $R_{\text {min }}$, occurs at I, the intersection between line FP and the aperture [27]. This point divides the aperture into two smaller convex arcs. On each arc, aperture elements have distances $R$ monotonically increasing from $R_{\min }$ to $R_{1}$ or $R_{2}$. The integral for calculating $\partial h\left(\mathbf{x}_{p}, t\right) / \partial t$ is taken over these two arcs separately. Eventually, we obtain the pressure field as

$$
\begin{aligned}
p\left(\mathbf{x}_{p}, t\right) & =\frac{\rho c \beta_{i}}{\pi} v\left(t-\frac{R_{\text {min }}}{c}\right) \\
& -\frac{\rho c}{2 \pi}\left[\beta_{1} v\left(t-\frac{R_{1}}{c}\right)+\beta_{2} v\left(t-\frac{R_{2}}{c}\right)\right],
\end{aligned}
$$

where $\beta_{i}$ is the angular coefficient corresponding to element at I. Thus, the pressure field has another pulse corresponding to $R_{\text {min }}$. With $R_{\text {min }}$ provided in Fig. 1(a), the time delay for this pulse is,

$$
\tau_{p, \min }=\frac{d-a}{c},
$$

where $a$ is the distance between $\mathrm{P}$ and $\mathrm{F}$. The expression is obtained after taking $\tau_{0}$ into account.

Similarly for $\mathrm{P}$ inside region (III) (Fig. 1(c)), where the aperture element at $\mathrm{I}$ has the maximal distance $R_{\max }$ to $\mathrm{P}$ [27]. The pressure field is given by,

$$
\begin{aligned}
p\left(\mathbf{x}_{p}, t\right) & =\frac{\rho c}{2 \pi}\left[\beta_{1} v\left(t-\frac{R_{1}}{c}\right)+\beta_{2} v\left(t-\frac{R_{2}}{c}\right)\right] \\
& -\frac{\rho c \beta_{i}}{\pi} v\left(t-\frac{R_{\max }}{c}\right) .
\end{aligned}
$$

The third pulse now corresponds to $R_{\max }$, with $R_{\max }$ provided in Fig. 1(c). The time delay for this pulse is given by

$$
\tau_{p, \max }=\frac{d+a}{c} .
$$

Equations (9) and (11) are recognized as the transmit time delay calculations in the virtual source approach for imaging points in regions (I) and (III) [15]. Based on the spherical wave approximation in that study, we can make a conjecture that the pressure field is dominated by the pulses at $\tau_{p, \text { min }}$ and $\tau_{p, \max }$ in those corresponding regions. In region (II), the minimal and maximal distances from $\mathrm{P}$ to the aperture are $R_{1}$ and $R_{2}$. Thus, as $\mathrm{P}$ moves from (I) to (II), the pulse at $\tau_{p, \min }$ merges with the pulse at $\tau_{p, 1}$. And as $\mathrm{P}$ moves from (III) back to (II), the pulse at $\tau_{p, \max }$ merges with the pulse at $\tau_{p, 2}$.

The strength of this analysis is reduced in some extent because of the approximations and assumptions that we have had to make. In the next section we validate the analysis numerically by using Field II [20], [21] to simulate the pressure field of a transmitted beam.

\section{Simulated pressure waveform}

The parameters for the simulation are based on the ULA-OP system described in Section III-B. Figure 2(a) shows simulated pressure fields at three points corresponding to the points labelled $\mathrm{P}$ in each of the three Figs. 1(a)-(c). They are rescaled by the maximal magnitude of the field at the point in region (II). These points are on a scanline offset to one side (laterally) from the centre of the aperture.

For the simplest case of region (II), the pressure field has two pulses with similar magnitudes. In region (III), the field is dominated by the pulse at $\tau_{p, \max }$. There is a small preceded pulse that may contain both pulses at $\tau_{p, 1}$ and $\tau_{p, 2}$. In the region far from the subaperture, $R_{1}$ and $R_{2}$ are approximately the same. Thus, those pulses can be merged into one. The pressure field in the near-field is more complicated. It is dominated by the pulse at $\tau_{p, \min }$, and following by unwanted ringing signals. The magnitude of the pulses in region (II) is significantly smaller than in regions (III) and (I) that contain the main energy of the beam. The magnitude further decreases with increasing distance from the beam centreline.

These ringing artefacts are the major difference between pressure waveforms observed in the near-field and far-field regions. In [28], Insana et al. used the Fresnel approximation to relate the artefacts to a quadratic phase factor. This phase factor must be eliminated to achieve far-field (Fraunhofer) 


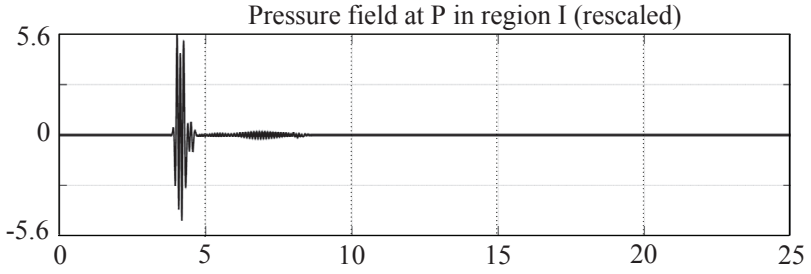

Pressure field at $\mathrm{P}$ in region II (normalized)
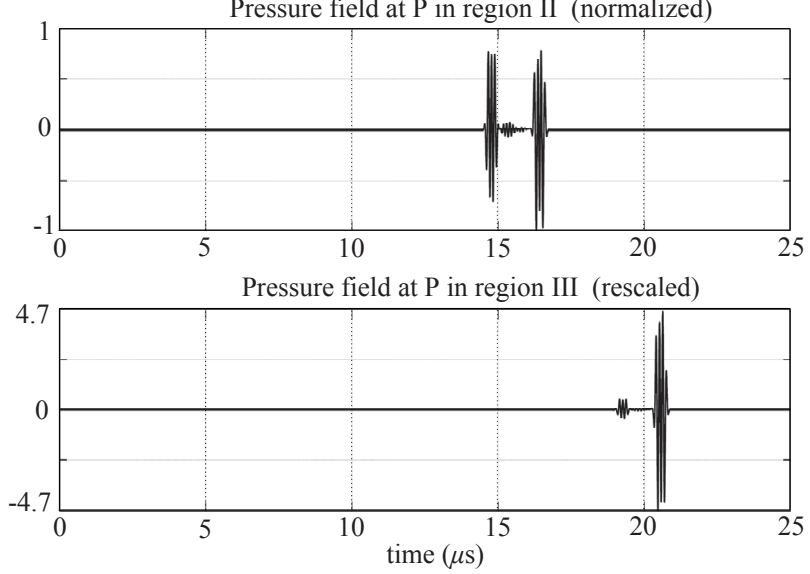

(a)

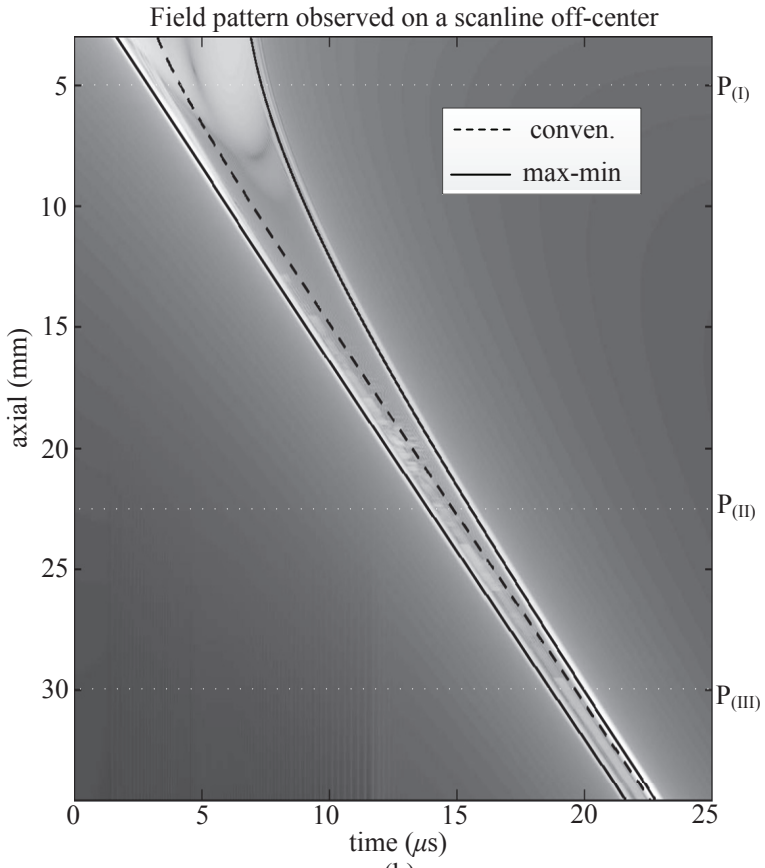

(b)

Fig. 2. (a) Simulations of the pressure field generated at the imaging point $P$ for the three cases depicted in Figs. 1(a), (b) and (c). The plots are rescaled by the maximal amplitude of the field in region (II). (b) Field patterns (envelope and log-compressed) formed by aligning the pressure fields generated at all points along the laterally offset scanline that passes through the three points labeled $\mathrm{P}$ in Figs. 1(a), (b) and (c). The envelope of pressure fields plotted in (a) are at positions indicated by $\mathrm{P}^{\prime} \mathrm{s}_{(\mathrm{I}-\mathrm{III})}$. On top of the pattern, time delay curves are calculated and plotted for two different strategies: the conventional approach (conven.); and from Eqs. (12) and (13) using the two-pulse characterization of the transmitted wave (max-min).

diffraction-limited spatial resolution. Although the artefacts are reduced by the delays associated with transmit focussing, they still affect the image quality in the near-field, as demonstrated in Section III. The ringing artefacts correspond to sidelobes in the transmit beamshape which can be suppressed using apodization (see [2], Figs. 2 and 3).

Because the ringing artefacts are small, the pressure field observed in the near-field is dominated by the pulse at $\tau_{p, \min }$. Thus, the pressure waveform can be characterized by the pulses at $\tau_{p, \text { min }}$ and $\tau_{p, \max }$ in regions (I) and (III) respectively. This illustrates one of the advantages of using the virtual source approach. Although the concept of the spherical wave is only approximated, the associated time delay calculations allow us to select signals with the highest amplitudes on each received waveform for data superposition. However, it also shows how the spherical wave assumption is violated in regions (II) and (IV) where the two pulses at $\tau_{p, 1}$ and $\tau_{p, 2}$ are comparable to each other. To utilize imaging data at pixels insides these regions, we first need to find a new way to describe the pressure field which is valid over the entire imaging region.

\section{Two-pulse characterization}

Instead of using the spherical wave approximation, we propose the use of two pulses to characterize the pressure field. They correspond to the maximal and minimal distances from $\mathrm{P}$ to the active aperture. Their time delays, $\tau_{p, \text { min }}$ and $\tau_{p, \max }$, are calculated based on a combination of Eqs. (7), (9), and (11). In particular, they are given by

$$
\begin{aligned}
\tau_{p, \text { min }} & =\frac{d-a}{c} \text { for } \mathrm{P} \text { in (I) } \\
\tau_{p, \text { min }} & =\frac{R_{1}}{c}-\tau_{0} \text { for } \mathrm{P} \text { in (II), (III) and (IV) }
\end{aligned}
$$

and

$$
\begin{aligned}
\tau_{p, \max } & =\frac{d+a}{c} \text { for } \mathrm{P} \text { in (III) } \\
\tau_{p, \max } & =\frac{R_{2}}{c}-\tau_{0} \text { for } \mathrm{P} \text { in (I), (II), and (IV). }
\end{aligned}
$$

The advantages of the two-pulse characterization can be demonstrated using a simulated field pattern on the axial distance-time axis. We first generate pressure fields for all points on the same scanline (through the three points labelled $\mathrm{P}$ in Figs. 1(a)-(c)) and align the signals together. We obtain a field pattern shown as a gray-scale image in Fig. 2(b) (after demodulated and log-compressed). The envelopes of the pressure fields plotted in (a) correspond to three horizontal lines at axial distances labelled $\mathrm{P}_{(\mathrm{I})}, \mathrm{P}_{(\mathrm{II})}$ and $\mathrm{P}_{(\mathrm{III})}$ down the right-hand side of Fig. 2(b). There are two white stripes across the pattern corresponding to the two pulses on each waveform. They indicate the times at which waves of useful amplitude reach each depth of this offset scanline. In the near-field, the unwanted ringing signals are strong and form the noticeable artefacts between the two stripes.

On top of the field pattern, we plot the transmit time delay curves calculated in the conventional approach (conven.), given by the first term on the right-hand side of Eq. (1). This time delay calculation is based on the condition that 
the pressure field is spherical and generated from the center of the transmit subaperture. The simulated results show that the (dashed) curve is always between the two high amplitude stripes. Hence, if we use this equation to calculate the time for the transmitted beam to reach the target, the selected data will contain mostly noise or ringing artefacts rather than useful information.

We next plot the time delay curves $\tau_{p, \min }$ and $\tau_{p, \max }$ given by Eqs. (12) and (13) applied to all points on the scanline (max-min). The two curves (solid lines) coincide with the white stripes in the image. These time delays allow us to extract the signal with highest energy in each echo RF trace. They apply to all pixels of the scanline, including those outside the limited angle. Thus, the time delays $\tau_{p, \min }$ and $\tau_{p, \max }$ can be used to calculate the time for the transmitted beam to reach the target. For a scan line at the centre of the beam (i.e. that passes through the focus), an equivalent figure would show the dotted and solid lines all coincident. Time delay calculation in Eq. (1) works fine along the centreline, as we would expect for the case of conventional dynamic focusing.

\section{E. Unified pixel-based beamforming}

In this new method, we use the proposed two-pulse characterization to design the transmit time delay in the beamforming calculation. The geometric optics approximation limits us to select only one data point on each received waveform for data superposition. While this is easy for imaging points in regions (I) and (III), where the waveform is dominated by pulses at $\tau_{p, \min }$ and $\tau_{p, \max }$ respectively, it presents a problem in (II) and (IV) where the two pulses at $\tau_{p, \min }$ and $\tau_{p, \max }$ (or $\tau_{p, 1}$ and $\tau_{p, 2}$ ) have similar magnitudes.

A key constraint for this calculation is a smooth time delay for the acoustic wave propagating from the source to individual imaging points. Because the sound-speed inside soft-tissue media varies within a small range, the transmit time delay or the travel time should not change abruptly when moving from one point to its neighbors. Any discontinuity in the travel-time field directly causes a discontinuity in the resulting image as data from one backscattered signal suddenly replaces different data over a short distance in the image. We therefore need to find a way of generating a consistent travel-time field such that $\tau_{p, \text { min }}$ is used as the transmit time in region (I), $\tau_{p, \max }$ in is used region (III) and there are no discontinuities in the transmit time field across the image.

First we note that there is no problem when regions (I) and (III) meet at the focus: $\tau_{p, \min }=\tau_{p, \max }$ at this point. Next, there is not much insonification at points in regions (II) and (IV) away from the focus. The backscattered signals from these points do not make much contribution the beamforming data summation; it is more important to guarantee the continuity of the transmit time field than to incorporate weak data backscattered far from the region covered by the beam. Our solution involves linearly interpolating the transmit time between region (I) and region (III) (across region (II) or (IV)), and masking the amplitude of the data from regions (II) and (IV) to ensure that the noise from these regions does not reduce the quality of the final image.
Let Fig. 1(b) be the geometry for calculating this time delay. We follow the scanline passing through $\mathrm{P}$ to find its intersections, $\mathrm{A}$ and $\mathrm{B}$, with the boundary of region (II). The time delays at $\mathrm{A}$ and $\mathrm{B}$ are given by $\tau_{a, \min }$ and $\tau_{a, \max }$ in Eqs. (9) and (11). At P, we calculate the time delay as

$$
\tau_{p}=\frac{\left|\mathbf{x}_{b}-\mathbf{x}_{p}\right|}{\left|\mathbf{x}_{b}-\mathbf{x}_{a}\right|} \tau_{p, 1}+\frac{\left|\mathbf{x}_{a}-\mathbf{x}_{p}\right|}{\left|\mathbf{x}_{a}-\mathbf{x}_{b}\right|} \tau_{p, 2}
$$

where $\mathbf{x}_{a}, \mathbf{x}_{b}, \mathbf{x}_{p}$ are vector positions of points $\mathrm{A}, \mathrm{B}$, and $\mathrm{P}, \tau_{p, 1}$ and $\tau_{p, 2}$ are given in Eq. (7). The receive time delay calculation (i.e. the time for the ultrasound wave to return from the scatterer to the probe) is given by the second term on the right-hand side of Eq. (1).

Data superposition still follows Eq. (2). However, we use the weighting coefficients $w_{i, j}$ to suppress backscattered noise at imaging points far from the focus as there is no risk of losing useful information. Thus for each particular transmit, we set the coefficient $w_{i, j}$ to one for all imaging pixels inside regions (I) and (III). Outside of those regions, the $w_{i, j}$ is regulated based on the width of the transmit beam in the lateral direction. In this study, we determine coefficient $w_{i, j}$ 's by using the simulated transmit beam. In general, measurements using hydrophone may be needed to determine the beamshape for this selection. This strategy combines the advantages of using a virtual source approach for imaging points in regions (I) and (III) with the optimal resolution of conventional PB beamforming around the focal depth. Therefore, we call it unified pixel-based beamforming.

\section{BEAMFORMER EVALUATION}

We demonstrate and evaluate four beamformers using both simulations and experiments. We use conventional dynamic focusing, conventional PB and unified PB beamformers developed in our studies, and the SA-BiPBF - the latest pixel-based focusing in the literature. The first three beamformers have a transmit focal depth of $20 \mathrm{~mm}$, in the middle of the imaging region, while the $\mathrm{SA}-\mathrm{BiPBF}$ has the beam focused at $40 \mathrm{~mm}$ outside the imaging region [16].

\section{A. Evaluation metrics}

We evaluate the beamformers based on the quality of image that each generates. Ultrasound image quality is usually measured using three metrics: spatial resolution, contrast resolution and penetration. The spatial resolution can be measured from the ultrasound speckle pattern [29], using

$$
S_{c}=\int_{-\infty}^{\infty} \frac{C_{X}(\mathbf{x})}{C_{X}(0)} d \mathbf{x},
$$

where $C_{X}(\mathbf{x})$ is the spatial auto-covariance function (ACF) for the RF data, and $d \mathbf{x}$ is the sampling interval 2-D vector. Equation (15) gives us the average coherence length of the speckle in the B-scan. Because pixel-based beamforming improves the information content of lateral scanlines, we are interested in the lateral $S_{c x}$ only. The smaller $S_{c x}$, the better the spatial resolution of the image.

The coherence length was developed based on a series of assumptions, including the presence of fully developed 

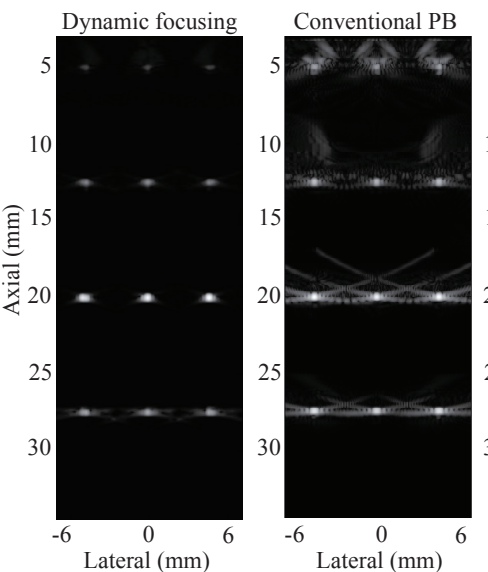

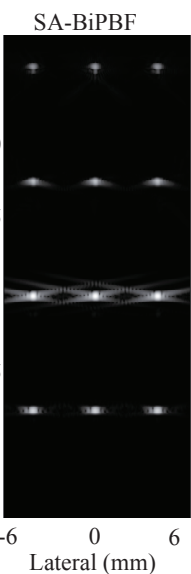

(a)
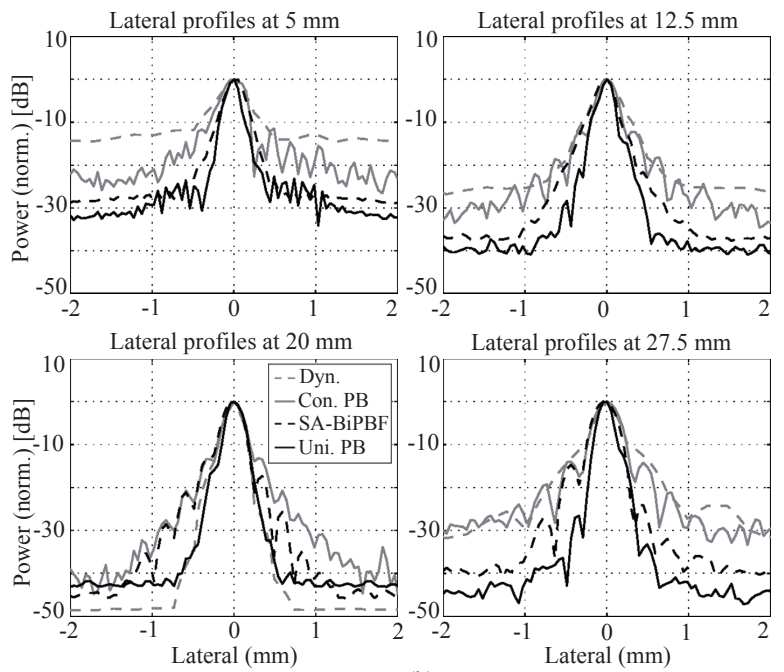

(b)

Fig. 3. (a) Images of twelve point targets generated with different beamformers: conventional dynamic focusing, conventional PB beamforming, synthetic aperture bi-directional pixel based focusing (SA-BiPBF) [16], and unified PB beamforming. All images are reconstructed from 64 transmits except for the dynamic focusing. They are log-compressed and displayed with a dynamic range of $50 \mathrm{~dB}$. (b) Lateral beam profiles of beamformers at depths of $5 \mathrm{~mm}$, $12.5 \mathrm{~mm}, 20 \mathrm{~mm}$, and $27.5 \mathrm{~mm}$ : conventional dynamic focusing (Dyn.), conventional PB beamforming (Con. PB), SA-BiPBF, and unified PB beamforming (Uni. PB). The legend in the plot for $20 \mathrm{~mm}$ is also relevant to the others.

speckle, no measurement noise, and a shift-invariant impulse response at the focal depth of the system [29]. In the nearfield where the data is affected by ringing artefacts, these assumptions are strongly violated. Therefore, we do not use this metric in the near-field for evaluating image quality.

The contrast resolution is usually measured using the contrast-to-noise ratio (CNR) between the lesion and background, where the speckle in the background is considered as noise. In our case, however, the background is not uniform and the variations correspond to meaningful signals indicating variations in scatterer density. Instead of using the CNR, we therefore evaluate the contrast resolution using the lateral response to simulated point targets generated by each beamformer.

Because the eSNR is depth-dependent, it can also be used to quantify the penetration. The eSNR (in $\mathrm{dB}$ ) is given by [30]

$$
\mathrm{eSNR}=10 \log _{10}\left(\frac{E_{h}}{\sigma_{n}^{2}}\right),
$$

where $E_{h}$ is the ensemble average energy of the echo beamformed signals and $\sigma_{n}^{2}$ is the noise power. To calculate the eSNR, we extract $E_{h}$ and $\sigma_{n}$ using the average/difference over several beamformed RF frames from the same sequence of scans. The data are extracted on a kernel that contains no specular reflector to avoid interferences from side-lobes in the noise power.

\section{B. System description and beamformer implementation}

In our study, the experimental data was acquired using an experimental ULA-OP ultrasound system developed in the Dipartimento di Elettronica e Telecomunicazioni at Università degli Studi di Firenze, Florence, Italy [22]. A linear array probe (LA523, Esaote spa, Florence, Italy) was used. This linear array has 192 elements each of dimensions $0.215 \times 6.0$ $\mathrm{mm}^{2}$ separated by a $0.03 \mathrm{~mm}$ kerf. There are 64 elements active in each transmit and receive cycle. Except at the edges of the image, the transmit focus is always on the centreline of the active aperture. The element pitch is $0.245 \mathrm{~mm}$. The echo data from all the receiving elements is stored in an array and can be individually accessed. In elevation, the aperture is weakly-focused using an acoustic lens with a focal length of $20 \mathrm{~mm}$. A three-cycle excitation voltage is applied to generate an ultrasound pulse with center frequency at $9.375 \mathrm{MHz}$ and an $85 \%$ pulse-echo bandwidth. The RF echo signals were sampled at $50 \mathrm{Msamples} / \mathrm{s}$, resulting in an axial sampling interval of $0.0154 \mathrm{~mm}$. The ULA-OP stores 2048 samples of each receiver signal which covers data ranging from $3 \mathrm{~mm}$ to $34 \mathrm{~mm}$ in depth. In the lateral direction the beam is stepped by an element pitch to generate envelope data with lateral spacing $0.245 \mathrm{~mm}$. The final B-mode image is then upsampled laterally for display with a $0.0154 \times 0.05 \mathrm{~mm}$ pixel size.

All pixel-based beamformers combine data from 64 transmits. This number then is reduced for conventional PB when we find the noise and near-field artefacts degrade the image quality. The weighted coefficient $w_{i, j}$ manages the contribution of transmit $i$ to a particular imaging point $\mathrm{P}$. For the unified PB beamformer, we set $w_{i, j}$ equal to one for $\mathrm{P}$ inside regions (I) and (III). As $\mathrm{P}$ moves out of those regions laterally, $w_{i, j}$ remains one if $\mathrm{P}$ is still within a distance equal to one element pitch. It is then reduced linearly and dropped to zero when $\mathrm{P}$ reaches a distance of three pitches. Further away, $w_{i, j}$ is set to zero or the contribution of transmit $i$ to $\mathrm{P}$ is completely suppressed. This selection process may need to be adapted to suit other probe geometries or transmit sequences.

\section{Simulated point scatterers}

We first apply the beamformers to data simulated by Field II to evaluate their ability to resolve point targets. The data 


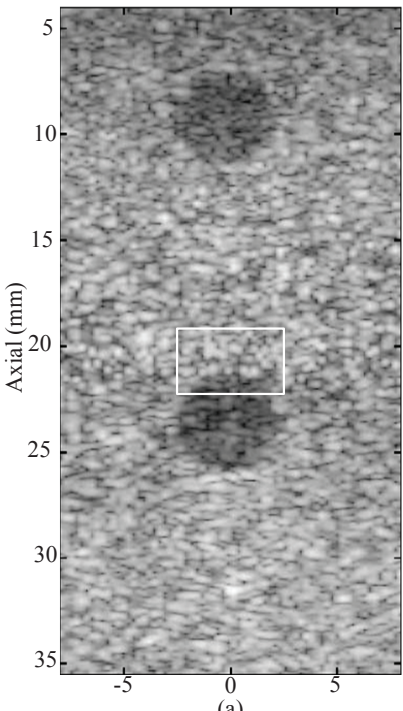

(a)

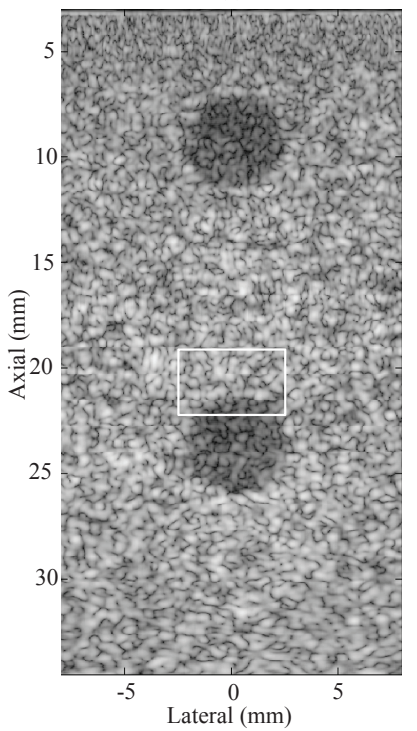

(d)

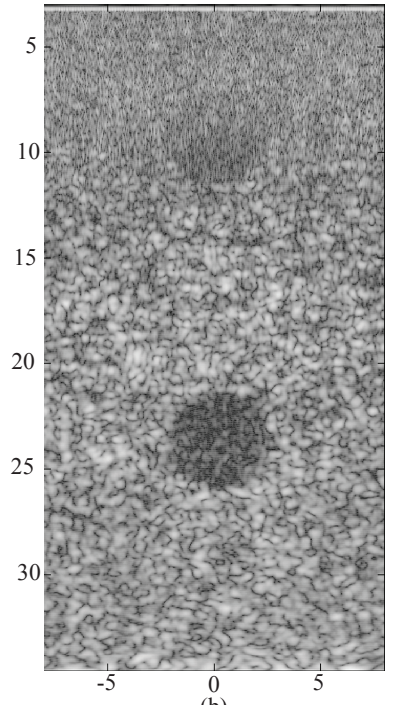

(b)

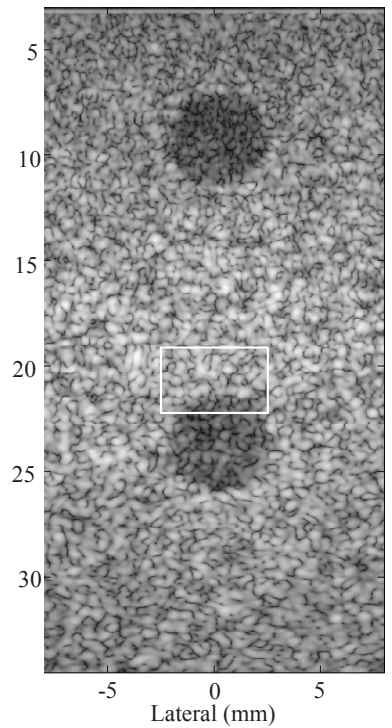

(e)

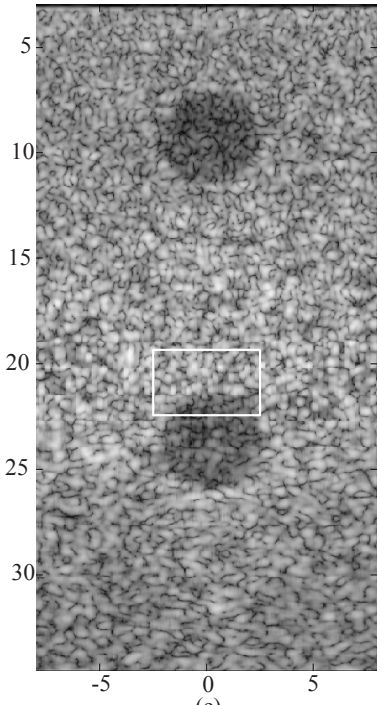

(c)

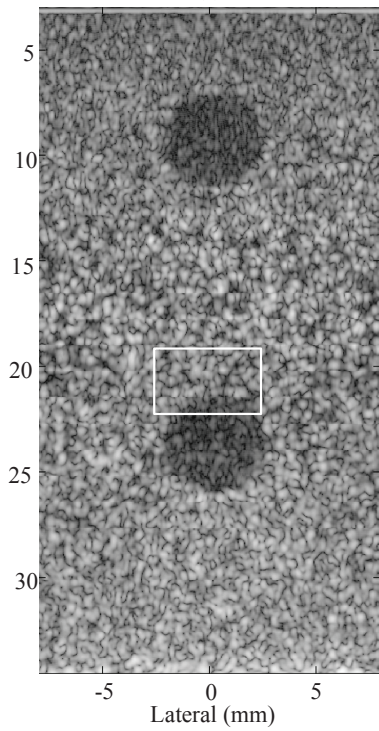

(f)

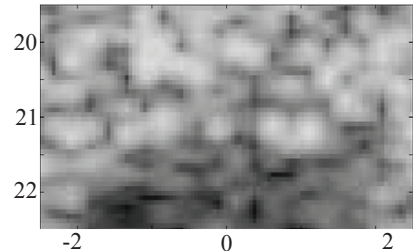

(a)

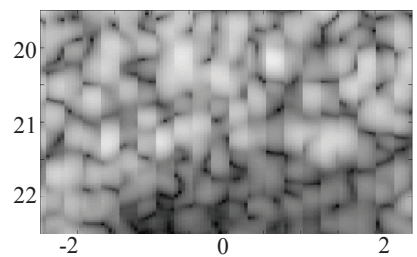

(c)

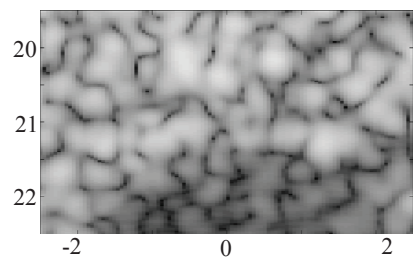

(d)

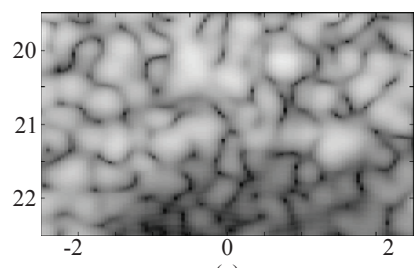

(e)

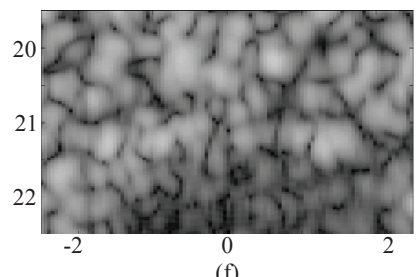

Fig. 4. Images of two idealised anechoic lesions generated with different beamformers and strategies: (a) conventional dynamic focusing, (b) conventional PB beamforming with $N_{t}=64$, (c) conventional PB beamforming with $N_{t}=2$, (d) conventional PB beamforming with $N_{t}=8$, (e) SA-BiPBF with $N_{t}=64$, and (f) unified PB beamforming with $N_{t}=64$. The regions enclosed by the white rectangles in (a), (c), (d), (e) and (f) are magnified and shown in the right-hand column. All images are log-compressed and displayed with a dynamic range of $50 \mathrm{~dB}$.

is generated with parameters matching the ULA-OP system. There are twelve points in the imaging field, distributed at four different depths. They are ranged from $5 \mathrm{~mm}$ to $27.5 \mathrm{~mm}$ with a $7.5 \mathrm{~mm}$ separation. There are two further columns with the same distribution located at $-4 \mathrm{~mm}$ and $4 \mathrm{~mm}$ in the lateral direction.

The generated images are shown in Fig. 3(a) (logcompressed). All pixel-based images are based on data from 64 transmits. The dynamic focused image shows bright and focused targets only at the focal depth. Visually, the unified PB has the best performance as all points are displayed with the smallest and most uniform appearance over all the depths. The other two, conventional PB and SA-BiPBF, have performance on par with each other. The conventional PB image shows evidence of the ringing artefacts in the near field. On the SABiPBF image, there are strong sidelobes observed at a depth of $20 \mathrm{~mm}$. They are caused by the focal depth of $20 \mathrm{~mm}$ in the elevational direction fixed by the acoustic lens. At this depth, the beam is broad laterally but highly focused in elevation. These artefacts therefore do not indicate an intrinsic limitation of the SA-BiPBF algorithm. The question of how to design elevational focussing to work best with SA-BiPBF is beyond the scope of the current paper.

The lateral beam patterns at different depths are plotted in Fig. 3(b) for the central targets. From this perspective, the unified PB shows the best overall spatial and contrast resolution. Dynamic focusing performs on par with the unified PB beamformer at the focal depth. In the other locations, it has widest mainlobes and highest sidelobes. The conventional PB and SA-BiPBF beam profiles have mainlobes approximately the same, however, the SA-BiPBF has lower sidelobes which indicates better contrast resolution. 


\section{Phantom study}

The beamformers are implemented experimentally with data acquired from the ULA-OP system by scanning a tissuemimicking phantom (manufactured by the Department of Medical Physics, University of Wisconsin, Madison, WI, USA). The manufacturer-reported speeds of sound ranging from $1538 \mathrm{~m} / \mathrm{s}$ to $1551 \mathrm{~m} / \mathrm{s}$ and the attenuation coefficient slope is $0.7 \mathrm{~dB} \mathrm{~cm}^{-1} \mathrm{MHz}^{-1}$. The two lesions being scanned are anechoic circular targets, $5 \mathrm{~mm}$ in diameter, positioned around depths of $9 \mathrm{~mm}$ and $24 \mathrm{~mm}$. There is no transmit apodization. On receive, the active aperture is varied with the depth to maintain a constant $f$ number of 1.25 . Imaging results are shown in Fig. 4(a)-(f). The right-hand column displays magnified views of regions enclosed by white rectangles on main images (a), (c), (d), (e) and (f) for clearer observation.

The dynamic focused image is extracted directly from the system and displayed in Fig. 4(a). The PB beamformers are then applied to pre-beamformed RF data from the same scanning process. Figure 4(b) shows an image generated using the conventional PB beamformer with number of transmits $N_{t}=64$. The speckle size is visually reduced in the focal and far-field regions yet there are strong near-field artefacts that degrade the appearance of the top lesion. These artefacts were also observed in [31] for a plane-wave transmission with a large subaperture. We show in our analysis they are linked to the effect of the quadratic phase factor [28].

We avoid these artefacts by limiting the data used in the superposition to a smaller number of transmits near to the beam center. We start with $N_{t}=2$ to generate the conventional PB image shown in Fig. 4(c). It has improvements over dynamic focusing in terms of speckle size and the shapes of both lesions, but there are block artefacts in the focal region shown in the magnified view. By increasing $N_{t}$, the block artefacts are reduced but this also introduces more near-field artefacts. Fig. 4(d) shows an image generated with $N_{t}=8$, selected by balancing the two sources of error. Its magnified view shows a smooth region and much reduced blurring comparing to the dynamic focused image in (a).

We apply the SA-BiPBF and the unified PB beamformers and show the results in Figs. 4(e)-(f), respectively. The magnified view in (f) shows a continuous region around the focal depth, indicating that artefacts associated with the virtual source are compensated completely. The image in (f) also has the finest speckle compared to the others in the figure. Images shown in (a), (d), (e), and (f) are then evaluated quantitatively using the coherence length and eSNR. The results are summarized in Table I. Strictly speaking, the denotation of focal and far-field on the table is not accurate for the SA-BiPBF as it has a focal length of $40 \mathrm{~mm}$, below the bottom of the image. However, its coherence lengths are still measured at the same depths as for the other beamformers.

In terms of spatial resolution measured by coherence lengths, the experimental results shown an agreement with simulation. The unified PB has smallest coherent length while those from the conventional $\mathrm{PB}$ and SA-BiPBF are on par with each other. Compared to dynamic focusing, all PB beamformers show significant enhancements over the entire imaging region. At the focal depth, the improvement is less impressive. This can be explained by the optimal resolution of dynamic focusing in the focal region. The eSNR measurements show that the SA-BiPBF performs best and provides the most stable image. It is worth noting that SA-BiPBF has a different transmit configuration from all the other approaches to achieve the deeper transmit focus. The eSNR may benefit from this as it is likely to increase the insonification and reduce the noise level in the part of the image furthest from the probe. It is also interesting to note that the finer and more uniform speckle generated by the unified $\mathrm{PB}$ beamformer reduces the perceived brightness of the corresponding B-mode image.

Although this study does not concentrate on temporal resolution, we still measure the computational time for each beamformer and show the results in the last column of Table I. The execution times are all measured using Matlab (Mathworks Inc. Natick, MA, USA) on a desktop PC (Windows 7, 64-bit system, Intel@ Core $^{\mathrm{TM}}$ i7-4770, and $8 \mathrm{~Gb}$ Memory). Hence these figures are only approximate indications of the relative un-optimised computational load, distorted by the strengths and weaknesses of Matlab.

\section{E. In vivo study}

In order to illustrate the robustness of the algorithms to phase aberration and other complexities of in vivo data we used the ULA-OP system to scan the thenar eminence in the left hand of a 50 year-old volunteer. This data was obtained with appropriate ethical clearance and informed consent. As it is impossible to hold the probe absolutely still with a human subject, we can only compare algorithms that can be applied to a single acquired set of data. The comparison thus includes images from the PB beamformers developed in our study and dynamic focusing. The generated images are shown in the top row of Figs. 5. The bottom row displays the magnified view for regions enclosed by the white rectangles on the top.

In clinical practice, ultrasound imaging can be used to detect flexor pollicis longus injury. The normal flexor tendon (which is the case in our study) has an ultrasound appearance that is fairly uniformly hyperechoic. Any anechoic interruption in the tendon fibers may indicate a tendon disruption. The anechoic area may represent blood or granular tissues where the tendon fibers are damaged [32].

The first half of the images is dominated by the abductor pollicis brevis (APB) muscle with a normal flexor pollicis longus (FPL) tendon traversing it. Between images (a) and (b) generated with dynamic focusing and conventional PB beamforming, respectively, image (b) looks finer with better visualization of the ultrasound speckle. However, the boundary near the transducer surface between the ABS and the skin is not clear indicating the effect of the ringing artefacts. To some extent, these effects also degrade the heathy features of the flexor tendon. There are also block artefacts shown in the magnified view of the image. Although these artefacts can be reduced by increasing the number of transmits, this also increases near-field artefacts.

Figure 5(c) shows imaging results with unified PB beamforming. Clearly, the healthy features of the flexor tendon are 
TABLE I

PERFORMANCE METRICS FOR BEAMFORMERS ON EXPERIMENTAL DATA ACQUIRED BY SCANNING A TISSUE-MIMICKING PHANTOM

\begin{tabular}{cccccc}
\hline \hline Beamformers & $\begin{array}{c}S_{c x} \\
\text { (focal) }\end{array}$ & $\begin{array}{c}S_{c x} \\
\text { (far-field) }\end{array}$ & $\begin{array}{c}\text { eSNR } \\
\text { (near-field) }\end{array}$ & $\begin{array}{c}\text { eSNR } \\
\text { (far-field) }\end{array}$ & $\begin{array}{c}\text { Computational } \\
\text { time }\end{array}$ \\
\hline Dynamic focusing & $0.40 \pm 0.005 \mathrm{~mm}$ & $0.61 \pm 0.015 \mathrm{~mm}$ & $28.1 \pm 0.9 \mathrm{~dB}$ & $22.8 \pm 0.3 \mathrm{~dB}$ & $42 \mathrm{sec}$. \\
Conventional PB (with $\left.N_{t}=8\right)$ & $0.37 \pm 0.004 \mathrm{~mm}$ & $0.46 \pm 0.004 \mathrm{~mm}$ & $31.5 \pm 1.5 \mathrm{~dB}$ & $27.5 \pm 1.5 \mathrm{~dB}$ & $15 \mathrm{~min}$. \\
SA-BiPBF (with $\left.N_{t}=64\right)$ & $0.38 \pm 0.007 \mathrm{~mm}$ & $0.44 \pm 0.009 \mathrm{~mm}$ & $38.5 \pm 0.6 \mathrm{~dB}$ & $35.4 \pm 0.2 \mathrm{~dB}$ & $59 \mathrm{~min}$. \\
Unified PB (with $\left.N_{t}=64\right)$ & $0.31 \pm 0.005 \mathrm{~mm}$ & $0.34 \pm 0.010 \mathrm{~mm}$ & $34.5 \pm 1.7 \mathrm{~dB}$ & $28.5 \pm 1.2 \mathrm{~dB}$ & $145 \mathrm{~min}$. \\
\hline \hline
\end{tabular}
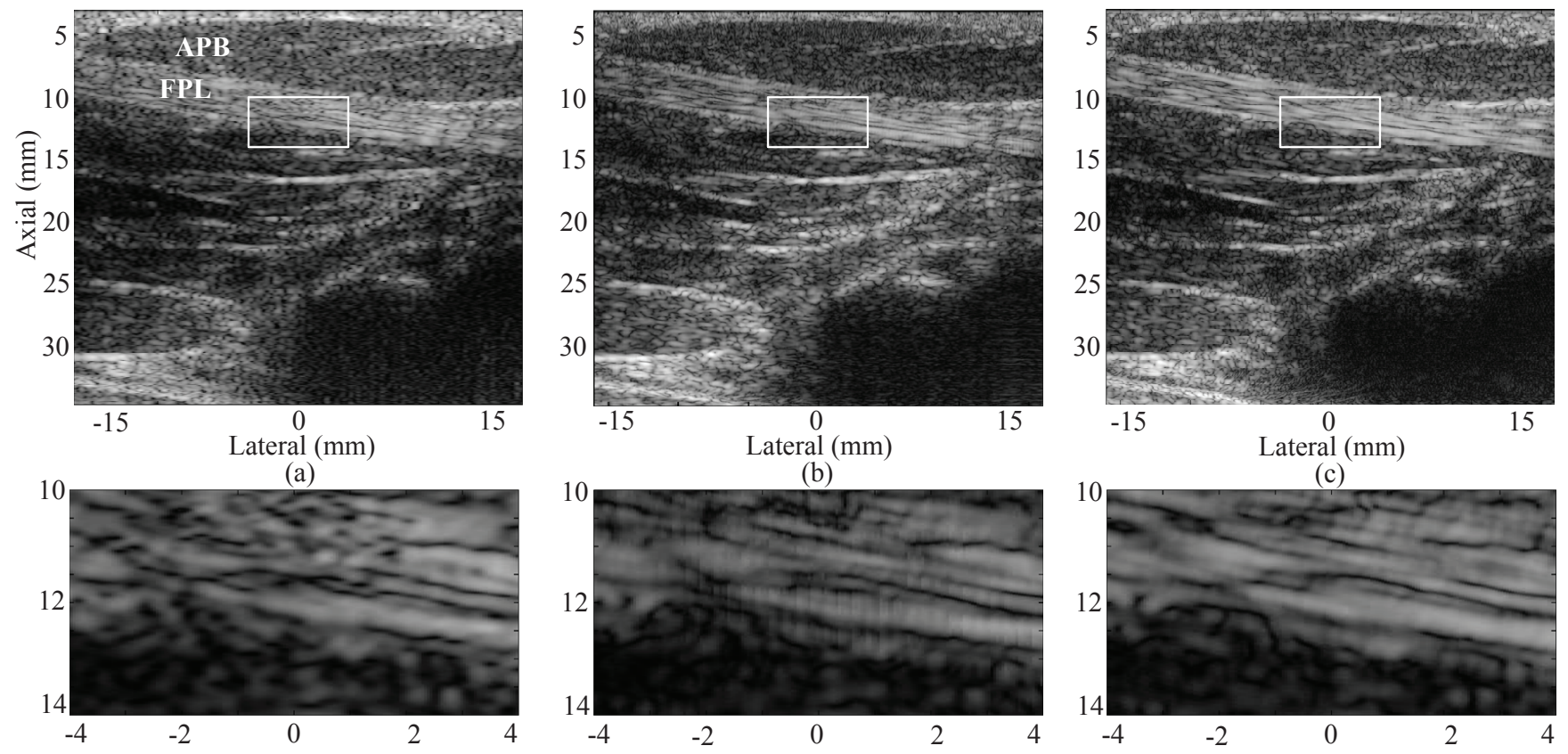

Fig. 5. (Top) B-mode images of the thenar eminence including a longitudinal view of the normal flexor pollicis longus (FPL) tendon, surrounded by the abductor pollicis brevis (APB) muscle. The images are generated and displayed using (a) dynamic focusing, (b) conventional PB beamforming with $N_{t}=8$, and (c) unified PB beamforming with $N_{t}=64$. Imaging data is acquired with a beam focused at $20 \mathrm{~mm}$. Labels in (a) are also relevant to the corresponding regions in images (b) and (c). (Bottom) Magnified views of the regions enclosed by the white rectangles in the top images. All images are log-compressed and displayed with a dynamic range of $50 \mathrm{~dB}$.

illustrated best as it is shown to be uniformly and brightly hyperechoic. There is also a better delineation of the muscle and tendon boundaries than in the other two images. In the magnified view, all blocking artefacts observed in the conventional $\mathrm{PB}$ view are eliminated. Compared to the dynamic focused magnified view in (a), the boundary between FPL and APB also shows up better with a more anechoic region for the APB muscles and a more uniformly hyperechoic region for the flexor tendon muscle. These enhancements illustrate the robustness of the unified $\mathrm{PB}$ beamforming algorithm in an in vivo context.

\section{Discussion}

The theoretical and experimental results all indicate that the conventional PB images should be formed from a limited number of transmits. Its time delay calculation, extended from the conventional dynamic scheme, only works effectively to generate scanlines around the beam center. This is similar to the way that the spherical wave assumption for the transmitted wave in SA imaging only can be made within a small angle
[4]. Consequently, the scanlines acquired at positions far from the principal axis make almost no positive contribution to the data superposition. Use of such offset scan lines in the nearfield adds unwanted ringing effects which degrade the shape of the top lesion (Fig. 4(b)). In the far-field, they enhance the spatial resolution giving a coherence length of $0.43 \pm 0.01 \mathrm{~mm}$, but decrease the eSNR to $20.7 \pm 0.1 \mathrm{~dB}$ which is even lower than that for dynamic focusing.

By using a small number of transmits $N_{t}=8$, the generated image (in Fig. 4(d)) has improvements in both spatial resolution and penetration over dynamic focussing. This strategy, however, is not the most effective exploitation of the echo signals in pre-beamformed data. For the image to be reconstructed with a maximal number of transmits the virtual source approach can be used to select appropriate data, even from scanlines far from the principal beam center.

We have studied two methods that incorporate a virtual source approach. The SA-BiPBF algorithm has a transmit beam focused deeper than the far edge of the imaging region to avoid artefacts from the discontinuity in the spherical wave 
approximation. SA-BiPBF has a spatial resolution on par with conventional PB beamforming $\left(N_{t}=8\right)$, indicating that the advantages of using the virtual source approach are reduced by the need for a broader transmit beam. The second approach was a new heuristic algorithm that we have called the unified pixel-based beamformer. This works with the beam focus at the normal depth. Simulations and experiments indicate that the unified PB can achieve a resolution in the near-field and far-field region almost as good as that at the focal depth. It combines the advantages of the virtual source approach in the near-field and far-field with good focal resolution as in the conventional approach.

\section{CONCLUSIONS}

We have shown in this study that transmit focusing has an important influence on image quality. In many variations of delay-and-sum beamforming the transmit beam is broadened to increase coherence among the echo signals. It is hard to compensate for this loss of transmit focusing during the receive process. The good performance of the unified PB beamformer in our study comes from a highly focused transmit beam and a dynamic time-delay computation to accumulate the bestpossible signals from the received waveforms at individual transducer elements.

While the unified pixel-based approach enhances the image quality at all depths, it has a particular impact in the nearfield. Echo data in this region is often blurred in existing methods through apodization to eliminate the ringing artefacts. In our approach however, this data is collected and utilized appropriately to improve the resolution of the resulting image. Thus the unified PB beamformer can provide clearer images with greater detail in the region near the transducer. Applications could include sonograms of relatively superficial tissue structures such as the tendon muscles illustrated in our in vivo study.

The geometrical optics approximation, on which the time delay calculations are based, limits us to use only one data point from each waveforms in the image reconstruction. We would like to incorporate more information from each waveform in the beamforming process. Using part of or even the full waveform could improve the image quality although it would also increase the implementation complexity. This remains an idea for future work. Meanwhile, the simplicity of the unified pixel-based beamformer, and its performance in both phantom and in vivo experiments, show the potential of the method for use in clinical applications.

\section{APPENDIX A: LIST OF ABBREVIATIONS}

$\begin{array}{ll}\text { APB } & \text { abductor pollicis brevis } \\ \text { ACF } & \text { auto-covariance function } \\ \text { BiPBF } & \text { bidirectional pixel-based focusing } \\ \text { SA-BiPBF } & \text { synthetic aperture BiPBF } \\ \text { DAS } & \text { delay-and-sum } \\ \text { eSNR } & \text { echo signal-to-noise ratio } \\ \text { CNR } & \text { contrast-to-noise ratio } \\ \text { FPL } & \text { flexor pollicis longus } \\ \text { PB } & \text { pixel-based }\end{array}$

RF

SA

ULA-OP

radio frequency

synthetic aperture

ultrasound advanced open platform

\section{ACKNOWLEDGMENT}

We thank Dr. Laurence Berman of Addenbrooke's Hospital, Cambridge, UK for performing the in vivo scan and advising on its interpretation. We are also grateful to the anonymous reviewers for their insightful comments.

\section{REFERENCES}

[1] N. Q. Nguyen, C. K. Abbey, and M. F. Insana, "An adaptive filter to approximate the Bayesian strategy for sonographic beamforming," IEEE Trans Med Imag, vol. 30, no. 1, pp. 28-37, 2011.

[2] K. E. Thomenius, "Evolution of ultrasound beamformers," Proceedings of the IEEE Ultrasonics Symposium, pp. 1615-1622, 1996.

[3] T. L. Szabo, Diagnostic ultrasound imaging: inside out, 2nd ed. Boston, MA: Elsevier Science, 2014.

[4] J. A. Jensen, S. I. Nikolov, K. L. Gammelmark, and M. H. Pedersen, "Synthetic aperture ultrasound imaging," in Ultrasonics, vol. 44, suppl., pp. e5-e15, 2006.

[5] M. Karaman, P.-C. Li, and M. O'Donnell, "Synthetic aperture imaging for small scale systems," in IEEE Trans Ultrason Ferroelec Freq Control, vol. 42, no. 3, pp. 429-442, 1995.

[6] K. L. Gammelmark and J. A. Jensen, "Multielement synthetic transmit aperture imaging using temporal encoding," in IEEE Trans Med Imag, vol. 22, no. 4, pp. 552-563, 2003.

[7] J. A. Jensen, O. Holm, L. J. Jensen, H. Bendsen, S. Nikolov, B. G. Tomov, P. Munk, M. Hansen, K. Salomonsen, J. Hansen, K. Gormsen, M. H. Pedersen, and G. K. Ammelmark, "Ultrasound research scanner for real-time synthetic aperture data acquisition," in IEEE Trans Ultrason Ferroelec Freq Control, vol. 52, no. 5, pp. 881-891, 2005.

[8] J. A. Jensen, H. Holten-Lund, R. T. Nilsson, M. Hansen, U. D. Larsen, R. P. Domsten, B. G. Tomov, M. B. Stuart, S. Nikolov, M. J. Pihl, Y. Du, J. H. Rasmussen, and M. F. Rasmussen,, "SARUS: A synthetic aperture real-time ultrasound system," in IEEE Trans Ultrason Ferroelec Freq Control, vol. 60, no. 9, pp. 1838-1852, 2013.

[9] D. P. Shattuck, M. D. Weinshenker, S. W. Smith and O. T. Von Ramm, "Explososcan: A parallel processing technique for high speed ultrasound imaging with linear phased arrays" in Journal of the Acoustical Society of America, vol. 75, no. 4, pp. 1273-1282, 1984.

[10] O. von Ramm, S. Smith and H. Pavy, "High-speed ultrasound volumetric imaging system. II. Parallel processing and image display" in IEEE Trans Ultrason Ferroelec Freq Control, vol. 38, no. 2, pp. 109-115, 1991.

[11] J. S. Hwang and T. K. Song, "A study of the display pixel-based focusing method in ultrasound imaging," in Ultrasonic Imaging, vol. 23 no. 1, pp. 1-18, 2001.

[12] R. E. Daigle, "Ultrasound imaging system with pixel oriented processing," U.S. Patent 2009/0112095 A1, May 19, 2009.

[13] Y. Lee, W. Y. Lee, C. E. Lim, J. H. Chang, T. K. Song, and Y. Yoo, "Compounded direct pixel beamforming for medical ultrasound imaging," in IEEE Trans Ultrason Ferroelec Freq Control, vol. 59, no. 3, pp. 573-582, 2012.

[14] J. N. Wright, S. H. Maslak, D. J. Finger, and A. Gee, "Method and apparatus for coherent image formation," U.S. Patent 5.623.928, 1997.

[15] M. H. Bae and M. K. Jeong, "A study of synthetic-aperture imaging with virtual source elements in B-mode ultrasound imaging systems," in IEEE Trans Ultrason Ferroelec Freq Control, vol. 47, no. 6, pp. 1510-1519, 2000

[16] C. Kim, C. Yoon, J. H. Park, Y. Lee, W. H. Kim, J. M. Chang, B. I. Choi, T. K. Song, and Y. M. Yoo, "Evaluation of ultrasound synthetic aperture imaging using bidirectional pixel-based focusing: preliminary phantom and in vivo breast study," in IEEE Trans Biomedical Engineering, vol. 60, no. 10, pp. 2716-2724, 2013.

[17] C. Passman and H. Ermert, "A 100-MHz ultrasound imaging system for dermatologic and ophthalmologic diagnostics," in IEEE Trans Ultrason Ferroelec Freq Control, vol. 43, no. 4, pp. 545-551, 1996.

[18] C. Frazer and W. D. O'Brien Jr., "Synthetic aperture techniques with a virtual source element," in IEEE Trans Ultrason Ferroelec Freq Control, vol. 45, no. 1, pp. 196-206, 1998. 
[19] H. Andresen, S. Nikolov, and J. A. Jensen, "Precise time-of-flight calculation for 3-D synthetic aperture focusing," in IEEE Trans Ultrason Ferroelec Freq Control, vol. 56, no. 9, pp. 1880-1887, 2009.

[20] J. A. Jensen and N. B. Svendsen, "Calculation of pressure fields from arbitrarily shaped, apodized, and excited ultrasound transducers," IEEE Trans Ultrason Ferroelec Freq Control, vol. 39, no. 2, pp. 262-267, 1992.

[21] J. A. Jensen, "Field: A program for simulating ultrasound systems," Med Biol Eng Comp, vol. 34, Supplement 1, Part 1, pp. 351-353, 1996.

[22] P. Tortoli, L. Bassi, A. Dallai, F. Guidi, and S. Ricci, "ULA-OP: An advanced open platform for ultrasound research," in IEEE Trans Ultrason Ferroelec Freq Control, vol. 56 no. 10, pp. 2207-2216, 2009.

[23] M. Born and E. Wolf, Principles of optics: electromagnetic theory of propagation, interference and diffraction of light. 7th ed. Cambridge, UK: Cambridge University Press, 1999.

[24] T. Xue, W. Lord, and S. Udpa, "Numerical analysis of the radiated fields of circular pistons and time-delay spehrically focused arrays," IEEE Trans Ultrason Ferroelec Freq Control, vol. 43, no. 1, pp. 7887, 1996.

[25] P. Crombie, P. A. J. Bascom, and R. S. C. Cobbold, "Calculating the pulsed response of linear arrays: accuracy versus computational efficiency," IEEE Trans Ultrason Ferroelec Freq Control, vol. 44, no. 5, pp. 997-1009, 1997.

[26] N. Denisenko, G. Scarano, M. Matteucci, and M. Pappalardo, "An approximate solution of the transient acoustic field," IEEE Trans Ultrason Ferroelec Freq Control, vol. SU-32, no. 6, pp. 821-827, 1985.

[27] R. S. C. Cobbold, Foundations of biomedical ultrasound. New York, NY: Oxford University Press, 2007.

[28] M. F. Insana, T. J. Hall, and L. T. Cook, "Backscatter coefficient estimation using array transducer," IEEE Trans Ultrason Ferroelec Freq Control, vol. 41, no. 5, pp. 714-723, 1994.

[29] R. F. Wagner, S. W. Smith, J. M. Sandrik, and H. Lopez, "Statistics of speckle in ultrasound B-scans," IEEE Trans Son Ultrason, vol. 30, no. 3, pp. 156-163, 1983.

[30] J. Liu, K.-S. Kim, and M. F. Insana, "SNR comparisons of beamforming strategies," in IEEE Trans Ultrason Ferroelec Freq Control, vol. 54, no. 5, pp. 1010-1017, 2007.

[31] M. Lewandowski, Z. Klimonda, P. Karwat, M. Seklewski, A. Nowicki, L. Bassi, and P. Tortoli, "Comparison of different schemes of synthetic transmit aperture using an ultrasound advanced open platform (ULAOP)," in Proceedings of the IEEE Ultrasonics Symposium, pp. 19881991, 2010.

[32] B. D. Fornage, Ultrasonography of muscles and tendons. New York, NY: Springer-Verlag, 1989. 